\title{
Dynamic effects of electromagnetic wave on a damped two-level atom
}

\author{
Z. Y. Zeng ${ }^{\ddagger}$, G. J. Zeng ${ }^{2}$, L. M. Kuang ${ }^{2}$, and L. D. Zhang ${ }^{1}$ \\ ${ }^{1}$ Institute of Solid State Physics, Chinese Academy of Sciences, P.O. Box 1129, Hefei 230031 \\ People's Republic of China \\ ${ }^{2}$ Department of Physics, Hunan Normal University, Changsha, Hunan 410081, People's Republic \\ of China
}

\begin{abstract}
We studied the dynamic effects of an electromagnetic(EM) wave with circular polarization on a two-level damped atom. The results demonstrate interesting ac Stark split of energy levels of damped atom. The split levels have different energies and lifetimes, both of which depend on the interaction and the damping rate of atom. When the frequency of the EM wave is tuned to satisfy the resonance condition in the strong coupling limit, the transition probability exhibits Rabi oscillation. Momentum transfer between atom and EM wave shows similar properties as the transition probability under resonance condition. For a damped atom interacting with EM field, there exists no longer stable state. More importantly, if the angular frequency of the EM wave is tuned the same as the atomic transition frequency and its amplitude is adjusted appropriately according to the damping coefficients, we can prepare a particular 'Dressed State' of the coupled system between atom and EM field and can keep the system coherently in this 'Dressed state' for a very long time. This opens another way to prepare coherent atomic states.
\end{abstract}

PACS numbers: 42.50.Vk, 32.80.-t, 03.65.Ge 


\section{Introduction}

It has been a long history for the study of dynamics of an atom in Electromagnetic (EM) field. In the past two decades, there has been revived and continuous interest to the subject of atomic motion in a Laser field, due to the variety of interesting and significant properties displayed in the interaction between atom and Laser field. These properties have been utilized to realize the deflection of atom beams by Lasers, (isotope separation) [1], Laser's cooling and trapping of atoms [2]. The successful observation [3] of the long-predicted Bose-Einstein condensation in 1995 was also attributed to the development of the technology of Laser's cooling and trapping of atoms.

The growing theoretical and experimental investigation has been devoted to the control of the lifetime of atoms in a cavity [4], since Purcell proposed the original idea that the lifetime of an atom can be modified [5]. These studies demonstrated that the spontaneous emission of atom can be suppressed or enhanced by a cavity [6], and thus the lifetime of atom can be controlled artificially. On the other hand, efforts to understand the spontaneous emission spectrum of two-level atoms when driven by a nearly resonant Laser field occupied central stage early on in the development of quantum optics. As is now well known, the primary spectral consequence of exposing a twolevel atom to a strong driving field is to split the undriven atomic emission into a triplet of peaks [7]. The atom and the driven field can be viewed as a single quantum system termed a Photonic Molecular, has eigenstates which has been referred to as dressed states [8]. As early as 1950s, Lamb et al. investigated the shortening of a metastable state caused by the coupling between an atom and a static electric field [9], and Bethe et al. studied such effects for a two-level atom [10]. These considerations, however, were limited to the static field and excluded the translational motion of the atom. Spontaneous emission by atom in free space and in cavity was studied recently in the framework of density matrix with the help of some approximations as Born-Markov approximation [11]. It claimed that spontaneous emission has no dependence on the translational motion of the atom in free space, since such dependence would lead to the violation of Galilean invariance [12]. Cavity mirrors break the Galilean invariance, so that atomic eternal motion effects are expected to exist in the spontaneous emission of the atom.

We studied the dynamic effects of a circular-polarized EM wave on a damped two-level atom, taking into consideration the center-of-mass motion of the atom. It was an extension of our previous paper concerning the non-damped atom in EM field [13]. The reason that we use circular-polarized EM wave is based on two facts: one is that a plane EM wave can be formed from two circularly polarized waves and vice versa, and the other that such formulation can voids the use of Rotating Wave Approximation (RWA). The procedure adopted is semi-classical, that is to say, the atom is treated as a quantum system while the electromagnetic wave is considered classically. Using such semi-classical procedure, the strict solution of Schrödinger equation for the damped two-level atom 
coupling with EM wave can be expected without any approximation except the dipole approximation, which is used in all the dipole-field interaction. In Sec. II, we derived the Schrödinger equations for the damped atom and obtained their strict solutions. Ac stark split of the unperturbed energy levels and the lifetimes of the atom in the four split levels were presented in Sec. III. In Sec. IV, we discussed the transition probabilities, finding that the coupling between atom and EM wave introduces into the stable state such as ground state part of the instability of the other states. When the frequency of the EM wave was tuned to satisfy resonance condition, the transition probability between two unperturbed states exhibits Rabi oscillation within a short period as in the static electric field case. In Sec. V we calculated the momentum transfer between the atom and the EM wave under the resonance condition with the consideration of some limiting cases. Sec. VI provided a new set of basis states-'dressed states', which is the superposition of the unperturbed basis states of the atom. In this 'dressed state' space, the total time-dependent Hamiltonian of the couple system can be diagonanized. If an EM wave is tuned the same frequency as the atomic transition frequency and its amplitude is adjusted appropriately according to the damping coefficients, then we can prepare a dressed state of very long lifetime at which the system can keep coherently for a quite long time. Summary along with concluding remarks was given in Sec. VI.

\section{Formulation Of The Problem}

We first proceed with a non-damped two-level atom of mass $m$, transition frequency $\omega_{a}$ and the dipole moment $\mathbf{D}$ interacting with a circular polarized EM wave propagating in the positive $z$ direction. The electric field $\mathbf{E}$ of the $E M$ wave is $\mathbf{E}=\left(E_{0} \cos \left(\omega_{L} t-k z\right),-E_{0} \sin \left(\omega_{L} t-k z\right)\right)$, where $E_{0}, \omega_{L}, k$ are the amplitude, angular frequency and wave vector respectively. In the dipole approximation, the Hamiltonian of a two-level atom in an EM wave is $H=P^{2} /(2 m)+H_{0}+H_{I}$, where $P^{2} /(2 m)$ is the kinetic energy term of the atom, $H_{0}=\operatorname{diag}\left(E_{1}, E_{2}\right)$ is the Hamiltonian associated with internal motion, and $H_{I}=-\mathbf{D} \cdot \mathbf{E}$ is the dipole interaction between atom and EM wave.

we choose the eigenstates $\mid \varphi_{1}>$ and $\mid \varphi_{2}>$ of the free Hamiltonian $H_{0}$ whose eigenvalues are, respectively, $E_{1}=\hbar \omega_{a} / 2$, and $E_{2}=-\hbar \omega_{a} / 2$ :

$$
\begin{aligned}
& H_{0}\left|\varphi_{1}>=E_{1}\right| \varphi_{1}>, \\
& H_{0}\left|\varphi_{2}>=E_{2}\right| \varphi_{2}>
\end{aligned}
$$

Then the dipole transition gives that,

$$
\begin{aligned}
& H_{I}^{i i}=<\varphi_{i}\left|H_{I}\right| \varphi_{i}>=0, \quad i=1,2 \\
& H_{I}^{21}=<\varphi_{2}\left|H_{I}\right| \varphi_{1}>=-\frac{1}{2} \hbar \Omega e^{-i\left(\omega_{L} t-k z\right)}, \\
& H_{I}^{12}=<\varphi_{1}\left|H_{I}\right| \varphi_{2}>=H_{I}^{21 *} \text {, }
\end{aligned}
$$


where $\Omega=2 D E_{0} / \hbar$. For a damped two-level atom, it can fall back to lower energy levels through spontaneous emission of one or several photons, with a probability $1 / \tau_{i}$ per unit time: $\tau_{i}$ is the lifetime of the unstable state $\left|\varphi_{i}\right\rangle$. If a state $\left|\varphi_{j}\right\rangle$ is non-damped (such as the ground state) in the absence of the coupling $H_{I}$, its lifetime $\tau_{j}$ is infinite. The Hamiltonian for the coupled system between a damped two-level atom and an EM wave can be readily obtained, by simply adding an imaginary term to the corresponding internal energy in the non-damped atom's Hamiltonian [13]: i. e., $E_{i}=E_{i}-i \hbar \gamma_{i} / 2$, where $\gamma_{i}=1 / \tau_{i}$ is the damping rate of the atom. It is noted that for the Hamiltonian of a damped atom in EM field, in which an imaginary term $i \hbar \gamma_{i} / 2$ exists, then it is not hermitian, and thus the probability to find the atom in all of its unperturbed states will not always equal to 1 . Such system is a dissipative system and its wave functions can not be normalized, and the total probability of finding the atom in its unperturbed states will not be conserved. By solving the Schrödinger equation $i \hbar \frac{d}{d t}|\Psi(t)>=H| \Psi(t)>$ in momentum space, where $\left|\Psi(t)>=\psi_{1}(t)\right| \varphi_{1}>+\psi_{2}(t) \mid \varphi_{2}>$, we get the following coupled equations in momentum space

$$
\begin{aligned}
i \hbar \frac{d}{d t} \psi_{1}(p, t) & =\left(\frac{p^{2}}{2 m}+E_{1}-i \hbar \gamma_{1} / 2\right) \psi_{1}(p, t)-\frac{1}{2} \hbar \Omega e^{-i \omega_{L} t} \psi_{2}(p-\hbar k, t), \\
i \hbar \frac{d}{d t} \psi_{2}(p, t) & =\left(\frac{p^{2}}{2 m}+E_{2}-i \hbar \gamma_{2} / 2\right) \psi_{2}(p, t)-\frac{1}{2} \hbar \Omega e^{i \omega_{L} t} \psi_{1}(p+\hbar k, t) .
\end{aligned}
$$

The coupled Schrödinger equations (3) can be decoupled and solved exactly[13]. Assuming that the damped atom is initially in the state $\mid \varphi_{2}>$ with momentum $p_{0}$, then $\psi_{2}(p, 0)=\delta\left(p-p_{0}\right)$, after some similar algebra as in Ref. 13, we obtain the following solutions

$$
\begin{aligned}
\psi_{1}(p+\hbar k, t) & =\delta\left(p-p_{0}\right) \frac{\Omega}{4 \beta(p)}\left[-e^{-i \omega_{1}^{+}(p) t}+e^{-i \omega_{1}^{-}(p) t}\right] \\
\psi_{2}(p, t) & =\delta\left(p-p_{0}\right)\left[\frac{\epsilon(p)+\beta(p)}{2 \beta(p)} e^{-i \omega_{2}^{+}(p) t}-\frac{\epsilon(p)-\beta(p)}{2 \beta(p)} e^{-i \omega_{2}^{-}(p) t}\right]
\end{aligned}
$$

which can be transformed into coordinate space

$$
\begin{aligned}
\phi_{1}(z, t)= & \frac{\Omega}{4(2 \pi \hbar)^{1 / 2} \beta\left(p_{0}\right)}\left\{-e^{\frac{i}{\hbar}\left[\left(p_{0}+\hbar k\right) z-E_{1}^{+}\left(p_{0}\right) t\right]}+e^{\frac{i}{\hbar}\left[\left(p_{0}+\hbar k\right) z-E_{1}^{-}\left(p_{0}\right) t\right]}\right\}, \\
\phi_{2}(z, t)= & \frac{1}{(2 \pi \hbar)^{1 / 2}}\left\{\frac{\epsilon\left(p_{0}\right)+\beta\left(p_{0}\right)}{2 \beta\left(p_{0}\right)} e^{\frac{i}{\hbar}\left[\left(p_{0}+\hbar k\right) z+E_{2}^{+}\left(p_{0}\right) t\right]}-\frac{\epsilon\left(p_{0}\right)-\beta\left(p_{0}\right)}{2 \beta\left(p_{0}\right)} \times\right. \\
& \left.e^{\frac{i}{\hbar}\left[\left(p_{0}+\hbar k\right) z+E_{2}^{+}\left(p_{0}\right) t\right]}\right\},
\end{aligned}
$$

where $\omega_{1}^{ \pm}(p)=\alpha_{1}(p) \pm \beta(p), \omega_{2}^{ \pm}(p)=\alpha_{2}(p) \pm \beta(p), E_{1}^{ \pm}=\hbar \omega_{1}^{ \pm}, E_{2}^{ \pm}=\hbar \omega_{2}^{ \pm}$, with

$$
\begin{aligned}
\alpha_{1}(p) & =\alpha_{2}(p)+\omega_{L}=\left(E_{+} / \hbar+\omega_{L}-i \gamma_{+}\right) / 2 \\
\epsilon(p) & =-\left(E_{-} / \hbar+\Delta \omega-i \gamma_{-}\right) / 2 \\
\beta(p) & =\sqrt{\epsilon^{2}(p)+\Omega^{2} / 4}
\end{aligned}
$$

with $E_{ \pm}=\frac{(p+\hbar k)^{2}}{2 m} \pm \frac{p^{2}}{2 m}, \Delta \omega=\omega_{a}-\omega_{L}$ and $\gamma_{ \pm}=\gamma_{1} \pm \gamma_{2}$ 
Though the solutions of Schrödinger equation for the damped atom have the same form as that for non-damped atom, the physical significance of these solutions are very different. The crucial discrepancy lies in that the coefficients and the exponential terms concerning the energy and lifetime in the solutions of Schrödinger equation for damped atom are complex function, while they are real function in that for non-damped atom. In addition, the wave functions of the atom can not be normalized, and the probabilities of finding the atom in its unperturbed states will be not always equal to 1.

\section{Ac Starc Split Of Energy Levels}

In the above section, we mentioned that the coefficients and the exponential terms related to the energy and the lifetime in the solutions of Schrödinger equation given by Eqn. (5) are both complex function. They can be decomposed into two parts-the real part designated by superscript $R$ concerning energy, and the imaginary part by superscript $I$ is related to damping rate. After some algebra, we have

$$
\begin{aligned}
E_{1}^{ \pm R} & =\left[E_{+}+\hbar \omega_{L} \pm 2 \hbar \beta^{R}(p)\right] / 2 \\
E_{2}^{ \pm R} & =\left[E_{+}-\hbar \omega_{L} \pm 2 \hbar \beta^{R}(p)\right] / 2 \\
E_{1}^{ \pm I} & =\hbar\left[\gamma_{+} \pm 2 \beta^{I}(p)\right] / 2 \\
E_{2}^{ \pm I} & =\hbar\left[\gamma_{+} \pm 2 \beta^{I}(p)\right] / 2
\end{aligned}
$$

where

$$
\begin{aligned}
\beta^{R}(p)= & \frac{\sqrt{2}}{4}\left\{\sqrt{\left[\left(E_{-} / \hbar+\Delta \omega\right)^{2}+\Omega^{2}-\gamma_{-}^{2}\right]^{2}+4 \gamma_{-}^{2}\left(E_{-} / \hbar+\Delta \omega\right)^{2}}+\right. \\
& \left.\left(E_{-} / \hbar+\Delta \omega\right)^{2}+\Omega^{2}-\gamma_{-}^{2}\right\}^{1 / 2}, \\
\beta^{I}(p)= & \frac{\sqrt{2}}{4}\left\{\sqrt{\left[\left(E_{-} / \hbar+\Delta \omega\right)^{2}+\Omega^{2}-\gamma_{-}^{2}\right]^{2}+4 \gamma_{-}^{2}\left(E_{-} / \hbar+\Delta \omega\right)^{2}}-\right. \\
& \left.\left(E_{-} / \hbar+\Delta \omega\right)^{2}-\Omega^{2}+\gamma_{-}^{2}\right\}^{1 / 2} .
\end{aligned}
$$

Inspecting Expressions (4) and (5), we find that both $\phi_{1}(z, t)$ and $\phi_{2}(z, t)$ comprise two plane waves, in which the energies and the lifetimes of the atom are different with the momenta being the same. Thus the two unperturbed levels of the atom are split into four levels of different energies, which are given by Eqn. (7). The energy difference $\Delta E=E_{1}^{+}-E_{1}^{-}=E_{2}^{+}-E_{2}^{-}=2 \hbar \beta^{R}\left(p_{0}\right)$ is the same for the two unperturbed levels of the atom. While the lifetimes(damping rate) of the atom in the four split-levels are obviously modified as compared with that in the unperturbed two states, though the imaginary part of the energy term can not be directly regarded as the inverse of the lifetime. In Fig. 1, we depicted the above energy level split caused by a time-varying EM field is referred to as ac Stark split.

\section{Transition Probabilities and Rabi Oscillation}


It is well known that, when a two-level atom is interacting with a static electric field, Rabi oscillation occurs, i. e., its transition probability will oscillates between the two-unperturbed states $\mid \varphi_{1}>$ and $\mid \varphi_{2}>$. From Eqn. (4) or (5), we can readily obtain the probabilities of finding the damped atom at time $t$ in its unperturbed state $\left|\varphi_{1}\right\rangle$ and $\left|\varphi_{2}\right\rangle$

$$
\begin{aligned}
\rho_{1}\left(p_{0}+\hbar k, t\right)= & \frac{\Omega}{8\left|\beta\left(p_{0}\right)\right|^{2}}\left\{\cosh \left[2 \beta^{I}\left(p_{0}\right) t\right]-\cos \left[2 \beta^{R}\left(p_{0}\right) t\right]\right\} e^{-\gamma+t}, \\
\rho_{2}\left(p_{0}, t\right)= & \frac{1}{2\left|\beta\left(p_{0}\right)\right|^{2}}\left\{\left[\left|\epsilon\left(p_{0}\right)\right|^{2}+\left|\beta\left(p_{0}\right)\right|^{2}\right] \cosh \left[2 \beta^{I}\left(p_{0}\right) t\right]+\left[\gamma_{-} \beta^{I}\left(p_{0}\right)-\left(E_{-} / \hbar+\Delta \omega\right)\right.\right. \\
& \left.\beta^{R}\left(p_{0}\right)\right] \sinh \left[2 \beta^{I}\left(p_{0}\right) t\right]+\left[\left|\beta\left(p_{0}\right)\right|^{2}-\left|\epsilon\left(p_{0}\right)\right|^{2}\right] \cos \left[2 \beta^{R}\left(p_{0}\right) t\right]-\frac{1}{2}\left[\gamma_{-} \beta^{R}\left(p_{0}\right)\right. \\
& \left.\left.+\left(E_{-} / \hbar+\Delta \omega\right) \beta^{I}\left(p_{0}\right)\right] \sin \left[2 \beta^{R}\left(p_{0}\right) t\right]\right\} e^{-\gamma_{+} t},
\end{aligned}
$$

Eqn. (9) demonstrated a fact that the probabilities of finding the atom in its unperturbed state $\mid \varphi_{1}>$ or $\mid \varphi_{2}>$ evolve with time in a quite complicated way. They depend not only on the amplitude and frequency of the coupling circular-polarized EM wave, also on the physical qualities of the atom such as dipole moment and kinetic energy. More importantly, the coupling of a two-level atom with a EM wave introduces into the stable state part of the instability of the other state. This can be verified as we set $\mid \varphi_{2}>$ to be a non-damped state $\left(\gamma_{2}=0\right)$, damped exponentials still exists in the probability to find the atom in this non-damped state $\left|\varphi_{2}\right\rangle$. Then we conclude that when a damped atom is exposing to an EM field, there exists no longer stable state for this atom. If the frequency of the EM is tuned such that $E_{-} / \hbar+\Delta \omega=0$ (which we defined as 'Resonance'), then the above probabilities have the following simple form

$$
\begin{aligned}
\rho_{1}\left(p_{0}+\hbar k, t\right)= & \frac{\Omega^{2}}{\Omega^{2}-\gamma_{-}^{2}} \sin ^{2}\left[\left(\Omega^{2}-\gamma_{-}^{2}\right)^{\frac{1}{2}} t / 2\right] e^{-\gamma_{+} t}, \\
\rho_{2}\left(p_{0}, t\right)= & \frac{\Omega^{2}}{\Omega^{2}-\gamma_{-}^{2}}\left\{\cos ^{2}\left[\left(\Omega^{2}-\gamma_{-}^{2}\right)^{\frac{1}{2}} t / 2\right]+\frac{\gamma_{-}\left(\Omega^{2}-\gamma_{-}^{2}\right)^{\frac{1}{2}}}{2 \Omega^{2}} \sin \left[\left(\Omega^{2}-\gamma_{-}^{2}\right)^{\frac{1}{2}} t\right]\right. \\
& \left.-\frac{\gamma_{-}^{2}}{\Omega^{2}}\right\} e^{-\gamma_{+} t} .
\end{aligned}
$$

for strong coupling $\left(\Omega>\gamma_{-}\right)$, while for for week coupling, i. e., $\Omega<\gamma_{-}$, we have

$$
\begin{aligned}
\rho_{1}\left(p_{0}+\hbar k, t\right)= & \frac{\Omega^{2}}{\gamma_{-}^{2}-\Omega^{2}} \sinh ^{2}\left[\left(\gamma_{-}^{2}-\Omega^{2}\right)^{\frac{1}{2}} t / 2\right] e^{-\gamma_{+} t} \\
\rho_{2}\left(p_{0}, t\right)= & \frac{\Omega^{2}}{\Omega^{2}-\gamma_{-}^{2}}\left\{\cosh ^{2}\left[\left(\gamma_{-}^{2}-\Omega^{2}\right)^{\frac{1}{2}} t / 2\right]+\frac{\gamma_{-}\left(\gamma_{-}^{2}-\Omega^{2}\right)^{\frac{1}{2}}}{2 \Omega^{2}} \sinh \left[\left(\gamma_{-}^{2}-\Omega^{2}\right)^{\frac{1}{2}} t\right]\right. \\
& \left.-\frac{\gamma_{-}^{2}}{\Omega^{2}}\right\} e^{-\gamma_{+} t} .
\end{aligned}
$$

The form of Eqn. (10) recalls Rabi' formula. According to the definition of $E_{-}$, 'Resonance' means that the frequency of EM wave equals to the energy difference including kinetic energy and internal energy of the two unperturbed basis states divided by $\hbar$. While in most literatures, resonance condition implies that the frequency of external field is tuned the same as the transition frequency 
between two states of atom. So the condition 'Resonance' used here includes the center-of-mass motion of the atom. When the coupling between atom and EM field is strong enough $\left(\Omega>\gamma_{-}\right)$, resonance condition $E_{-} / \hbar+\Delta \omega=0$ guarantees the atom to oscillate between the state $\mid \varphi_{1}>$ and $\mid \varphi_{2}>$ within a period $\Delta t<\tau_{+}=\frac{1}{\gamma_{+}}$. This can be clearly observed from Fig. 2. At times $t>\tau_{+}$, the atom will decayed into the lower energy states, since the probabilities of finding the atom in $\mid \varphi_{1}>$ and $\mid \varphi_{2}>$ decrease rapidly with time for $t>\tau_{+}$. Note that if the damping is excluded in our consideration, i. e., $\gamma_{-}=\gamma_{+}=0$, then we recover the sinusoidal oscillation as expressed in Eqn. 5 in Ref. 13. In addition, according to Eqn. (8), EM wave provides the atom with a certain probability to transit from the lower energy state $\mid \varphi_{1}>$ to the higher energy state $\left|\varphi_{2}\right\rangle$ by absorbing a or several photons of momenta $\hbar k$, before it decays to lower energy states. If the EM wave is switched off, then the atom will directly decay into other states than the unperturbed state $\left|\varphi_{2}\right\rangle$ and it is impossible to find the atom in the higher energy states. At $t=2 n \pi /\left(\Omega^{2}-\gamma_{-}^{2}\right)^{\frac{1}{2}}(n=0,1,2, \ldots)$, the probability of seeing the atom in state $\mid \varphi_{1}>$ is zero, and the probability to find the atom still in state $\mid \varphi_{2}>$ arrives maximum. For weak coupling, the probabilities of finding the atom in its unperturbed states is a sum of damped exponentials. This result has a simple physical interpretation: the lifetime $\tau_{+}=1 / \gamma_{+}$is so short that the system is damped into the lower energy states than $\mid \varphi_{2}>$ before the coupling has time to oscillate between states $\mid \varphi_{2}>$ and $\left|\varphi_{1}\right\rangle$.

\section{Momentum Transfer between atom and EM Wave}

In our previous work, we have shown that a circular-polarized EM wave exerts expelling or trapping force to a non-damped two-level atom, due to the momentum transfer between the atom and EM wave. The properties of the action depend on wether the atom motion direction is the same as or opposite to the propagating direction of EM wave, and the initial state of the non-damped atom. In this section, we studied the momentum transfer between a damped atom and a circularly polarized EM wave, and we only considered the case that the atom is initially in its undamped state $\mid \varphi_{2}>\left(\gamma_{2}=0\right)$. We also compared the results for damped atom with that for non-damped atom. Using the expression of probabilities to find the atom in its states $\mid \varphi_{1}>$ and $\mid \varphi_{2}>$, we have the average values of the momenta of the damped atom

$$
p_{a}=\left(p_{0}+\hbar k\right) \rho_{1}\left(p_{0}+\hbar k, t\right)+p_{0} \rho_{2}\left(p_{0}, t\right) .
$$

It is easy to show that the expressions of averaged momenta can be recovered into non-damped atom case in the absence of damping emission. If the averaged momenta of the atom is known, the momentum transfer between atom and EM wave are calculated to be

$$
\Delta p=p_{a}-p_{0}
$$

In the following, we discuss in detail the momentum transfer under the resonance condition. In the 
case of strong coupling, we have

$$
\Delta p=\left(e^{-\gamma_{1} t}-1\right) p_{0}+\frac{\hbar k \Omega^{2}}{\Omega^{2}-\gamma_{1}^{2}} \sin ^{2}\left[\left(\Omega^{2}-\gamma_{1}^{2}\right)^{\frac{1}{2}} t / 2\right] e^{-\gamma_{1} t}+p_{0} \frac{\gamma_{1}\left(\Omega^{2}-\gamma_{1}^{2}\right)^{\frac{1}{2}}}{2 \Omega^{2}} \sin \left[\left(\Omega^{2}-\gamma_{1}^{2}\right)^{\frac{1}{2}} t\right] e^{-\gamma_{1} t},
$$

for weak coupling, the momentum transfer is

$$
\Delta p=\left(e^{-\gamma_{1} t}-1\right) p_{0}+\frac{\hbar k \Omega^{2}}{\gamma_{1}^{2}-\Omega^{2}} \sinh ^{2}\left[\left(\gamma_{1}^{2}-\Omega^{2}\right)^{\frac{1}{2}} t / 2\right] e^{-\gamma_{1} t}+p_{0} \frac{\gamma_{1}\left(\gamma_{1}^{2}-\Omega^{2}\right)^{\frac{1}{2}}}{2 \Omega^{2}} \sinh \left[\left(\gamma_{1}^{2}-\Omega^{2}\right)^{\frac{1}{2}} t\right] e^{-\gamma_{1} t}
$$

The average force exerted to the damped atom by the EM wave can be also obtained from Ehrenfest's theorem: $F=\frac{d}{d t} \Delta p$. From Eqn. (16), for $\gamma_{1}<<\Omega$, we can approximate $e^{-\gamma_{1} t} \simeq 1$ and omit the first and third terms in Eqn. (16), then we recovered the results for non-damped atom: $\Delta p=\hbar k \sin ^{2}(\Omega t / 2)$ and $F=\Omega \hbar k \sin (\Omega t) / 2$. In this case, the action of the EM wave on the atom shows the expelling property, because the direction of the average momentum the atom requires from the EM is the same as the propagating direction of the EM wave. This is easily understood since the spontaneous emission has trivial effects on the momentum transfer. For $t<<\tau_{1}$, the results will be in good agreement with that in Ref. 1 . Let us consider another limiting case $\tau_{1}>>\Omega$, according to Eqn. (17), we can readily conclude that the momentum transfer in this limit tends to zero rapidly. This phenomenon can be understood easily since spontaneous emission induces no net momentum transfer. The most interesting is the intermediate case where spontaneous emission process takes its effects. As the transition probability, the momentum transfer is expected to exhibit interesting oscillation in the strong coupling limit and rapid damping for weak coupling, though it is positive in the two limits. When a damped atom is irradiated by an EM wave, absorption-emission processes proceed at two distince rates. Photons are absorbed from and emitted into the applied wave at the induced rate $\Omega$, and occasionally photons are spontaneous emitted in random directions at the spontaneous rate $\gamma_{1}$. In the strong coupling case that the induced rate exceeds the spontaneous rate, the momentum transfer operating at the induced rate will be more efficient and more rapid than that at spontaneous rate. Thus the momentum transfer will be similar to the case for non-damped atom, with a resultant positive momentum transfer for a short time showing expelling property and a fluctuating ( the third term in Eqn. (16) charactering the spontaneous process. While for weak coupling, the spontaneous rate exceeds the induced rate, then no net momentum transfer is expected at times $t>\tau_{1}$, since the spontaneous emission occurs in random directions and thus introduces no net momentum transfer.

\section{Diagonization of $\mathrm{H}(\mathrm{t})$ and 'Dressed States'}

From Eqns. (4) and (5) or following the relation (20) and (21) of Ref. 13 between the amplitudes $\psi_{1}(p, t)$ and $\psi_{2}(p, t)$, we can express the wave function in the form

$$
\Psi(P, t)=a_{1}\left|\varphi_{-}(t)>e^{-i\left[\frac{E_{+}}{2 \hbar}+\beta^{R}(p)\right]} e^{-\left[\frac{\gamma_{+}}{2}-\beta^{I}(p)\right] t}+a_{2}\right| \varphi_{+}(t)>e^{-i\left[\frac{E_{+}}{2 \hbar}-\beta^{R}(p)\right]} e^{-\left[\frac{\gamma_{+}}{2}+\beta^{I}(p)\right] t} .
$$


where we defined a set of new time-dependent basis states $\mid \varphi_{-}(t)>$ and $\mid \varphi_{+}(t)>$, which are the superposition of the unperturbed basis states $\mid \varphi_{1}>$ and $\left|\varphi_{2}\right\rangle$

$$
\begin{aligned}
& \left|\varphi_{-}(t)>=\cos \left(\vartheta_{-}\right) e^{-i \omega_{L} t / 2} e^{i \theta_{-}}\right| \varphi_{1}>+\sin \left(\vartheta_{-}\right) e^{i \omega_{L} t / 2} \mid \varphi_{2}> \\
& \left|\varphi_{+}(t)>=\cos \left(\vartheta_{+}\right) e^{-i \omega_{L} t / 2} e^{i \theta_{+}}\right| \varphi_{1}>+\sin \left(\vartheta_{+}\right) e^{i \omega_{L} t / 2} \mid \varphi_{2}>
\end{aligned}
$$

with $\vartheta_{\mp}=\operatorname{Arctg}\left(\frac{\Omega}{2|\epsilon(p) \mp \beta(p)|}\right)$ and $\theta_{\mp}=\operatorname{Arctg}\left(\frac{\epsilon^{I}(p) \mp \beta^{I}(p)}{\epsilon^{R}(p) \mp \beta^{R}(p)}\right)$, where $\beta^{R}(p)$ and $\beta^{I}(p)$ are given by expression (8). In the $\left\{\left|\varphi_{-}(t)>,\right| \varphi_{+}(t)>\right\}$ basis, the total Hamiltonian $H(t)$ can be written as

$$
H=\left[\begin{array}{cc}
\frac{E_{+}}{2}+\hbar \beta^{R}(p)+i \hbar\left(\gamma_{+} / 2-\beta^{I}(p)\right) & 0 \\
0 & \frac{E_{+}}{2}-\hbar \beta^{R}(p)+i \hbar\left(\gamma_{+} / 2+\beta^{I}(p)\right)
\end{array}\right]
$$

Thus, in the $\left\{\left|\varphi_{-}(t)>,\right| \varphi_{+}(t)>\right\}$ space, the total Hamiltonian of the system is diagonanized. The basis states $\left\{\left|\varphi_{-}(t)>,\right| \varphi_{+}(t)>\right\}$ should be regarded as the basis states belonging to the coupled system between atom and EM wave, rather than the basis states of the damped atom, since $\mid \varphi_{-}(t)>$ and $\mid \varphi_{+}(t)>$ include the contributions of the EM wave with circular polarization, as can be seen from Eqn. (15). In this sense, we call the states $\left\{\left|\varphi_{-}(t)>,\right| \varphi_{+}(t)>\right\}$ dressed states belonging to the coupled system, of which the energies and the lifetimes are

$$
\begin{aligned}
E_{\varphi_{\mp}} & =\frac{1}{2}\left[\frac{(p+\hbar k)^{2}}{2 m}+\frac{p^{2}}{2 m} \pm \hbar \beta_{R}(p)\right] \\
\tau_{\varphi_{\mp}} & =\frac{1}{\gamma_{+} \mp 2 \beta^{I}(p)} .
\end{aligned}
$$

For the above 'Dress states', their lifetimes contain two parts, one of which is related to the lifetimes of the atom in its unperturbed states, while another depends on the interaction between atom and EM wave. If we adjust the interaction between atom and EM field, we can effectively elongate the lifetime of the coupled system in the state $\left|\varphi_{-}(t)\right\rangle$ and shorten the lifetime in $\left|\varphi_{+}(t)\right\rangle$. In other words, if an damped two-level atom is initially prepared in the dressed state $\mid \varphi_{-}(t)>$, it would stay coherently in this state for a longer time than $\tau_{\varphi_{+}}$, since the spontaneous emission can be suppressed to a great extent. This opens another way to prepare coherent atomic states.

Under the condition of resonance we have

$$
\begin{aligned}
E_{\varphi_{\mp}} & =\frac{1}{2}\left[\frac{(p+\hbar k)^{2}}{2 m}+\frac{p^{2}}{2 m} \pm \frac{\hbar\left(\Omega^{2}-\gamma_{-}^{2}\right)^{\frac{1}{2}}}{2}\right] \\
\tau_{\varphi_{\mp}} & =\frac{1}{\gamma_{+}}
\end{aligned}
$$

for strong coupling $\left(\Omega>\gamma_{-}\right)$, and

$$
\begin{aligned}
E_{\varphi_{\mp}} & =\frac{1}{2}\left[\frac{(p+\hbar k)^{2}}{2 m}+\frac{p^{2}}{2 m}\right], \\
\tau_{\varphi_{\mp}} & =\frac{1}{\gamma_{+} \mp\left(\gamma_{-}^{2}-\Omega^{2}\right)^{\frac{1}{2}}}
\end{aligned}
$$


for weak coupling $\left(\Omega<\gamma_{-}\right)$.

From Eqns. (22) and (23), one can find that, if the EM wave is tuned such that $E_{-} / \hbar+\Delta \omega=0$ and the coupling is strong enough $\left(\Omega>\gamma_{-}\right)$, then the dress states $\mid \varphi_{-}(t)>$ and $\left|\varphi_{+}(t)\right\rangle$ have different energies and the same lifetime; in the weak coupling case $\left(\Omega<\gamma_{-}\right)$, the energies are the same and the lifetimes are different, this is to say, for weak coupling, there exists degenerate dressed states for the coupled system.

As a concrete example, we considered a moderately massive atom of mass $m \simeq 10^{-23} \mathrm{G}$, transition frequency $\omega_{a} \simeq 10^{-16} \mathrm{sec}^{-1}$, original velocity $v_{0} \simeq 20 \mathrm{msec}^{-1}$, lifetime of the excited state $\tau_{1} \simeq 10^{-9} \sim 10^{-6} \mathrm{sec}$, dipole momentum $D \simeq 1.6 \times 10^{-29} \mathrm{Cm}$ and a circular polarized EM wave of angular frequency $\omega_{L} \simeq \omega_{a} \simeq 10^{16} \mathrm{sec}^{-1}$. Since $p_{0} \simeq 2 \times 10^{-19} \mathrm{Nsec}<<m c \simeq 3 \times 10^{-12}$ $\mathrm{N} \mathrm{sec}^{-1}$, the resonance condition is usually satisfied. To prolong the lifetime of the dressed state $\mid \varphi_{-}(t)>$, the amplitude of circular-polarized EM field should be small enough and must be less than the $10^{3} \sim 1 \mathrm{Vm}^{-1}$. Therefore if we want to prepare the dressed state mentioned above at which the coupled system can keep for a long time, we may irradiate the atom with a weak circularpolarized EM wave of the same angular frequency as the atomic transition frequency. The choice of the amplitude of EM wave depends on the lifetimes (damping coefficients) of the damped atom.

\section{Summary}

We have studied the dynamic effects of a circular-polarized EM wave on a damped tow-level atom, with the consideration of the center-of-mass motion of the atom. Schrödinger equation of the system and their strict solutions were given in Sec.II. It is shown that, two unperturbed levels of the atom is split into four levels of different energy and lifetime by the coupling between the atom and EM wave. We found that the energies and lifetimes of the four split-levels depend not only on the strength and frequency of the EM wave, but also on the kinetic energy,damping rate and dipole moment of the damped atom. The analytical relation between them was also presented.

EM wave can introduces into the non-damped state as ground state part of the instability of the other states, hence no stable state exists for a damped atom interacting with EM wave. When the frequency of the EM wave is tuned to satisfy the resonance condition, Rabi oscillation occurs in the transition probability for strong coupling, and rapid damping happens for weak coupling.

Under resonance condition, momentum transfer between atom and EM wave shows interesting oscillating in the strong coupling case and rapid damping in the weak coupling case as transition probability, which depends on wether the induced emission-absorption rate or spontaneous rate exceeds the other. For $\gamma_{1}<<\Omega$, the momentum transfer for damped atom is very similar to that for non-damped atom, while for $\gamma_{1}>>\Omega$, the momentum transfer will tends to zero very rapidly.

We also defined a new set of basis states-dressed states, which is the superposition of the unperturbed states and will evolve with time. In this new set of basis states, the total time-dependent 
Hamiltonian of the coupled system between the damped atom and EM wave is diagonanized and will be independent of time. Moreover, the energies and lifetimes of the new basis states can be effectively controlled. Thus the system can be coherently stay in one of the dressed states for a very long time, if the circular-polarized EM wave is tuned the same frequency and its amplitude is made appropriate according to the atomic damping coefficients. This opens another way to prepare coherent atomic states.

It is interesting to compare the results with that without the consideration of damping in Ref. 13, which studied the level split and momentum transfer between the atom and EM field, showing that the trapping or expelling force of EM wave on atom depends on the initial state of the atom. However, if spontaneous emission is taken into consideration, the energies of the split-levels will be related to the damping, and the probability of finding the atom in its all unperturbed states will not equal to 1 as for non-damped atom. The momentum transfer is also related to the damping and the results for non-damped atom can be recovered as $\gamma_{-}<<\Omega$. Within a short period, the motion of the atom will be the same (sinusoidal oscillation) both for damped and for non-damped atom. In addition, the problem for damped atom is more practicable. Non-damped atom is only a particular case of damped atom. For damped atom, we can prepare a special state, in which the atom would stay coherently for a long time by the effective adjusting of the coupling.

Due to the similarity between the quantum dots and atoms [14], some results in this paper can be directly applicable to the coupling of the quantum dots with an electromagnetic wave.

\section{References}

†: zyzeng@mail.issp.ac.cn

1. R. J. Cook and A. F. Bernhardt, Phys. Rev. A 18, 2533 (1978).

2. J. Dalibard and C. Cohen-Tannoudji, J. Opt. Soc. Am. B 6, 2023 (1989).

3. M. H. Anderson et al., Science, 269, 198 (1995)

4. P. R. Berman, Cavity Quantum Electrodynamics (Academic press, INC, Harcourt Brace \& Company, 1994).

5. E. M. purcell, phys. Rev. 69, 681 (1946).

6. D. Kleppner, Phys. Rev. Lett. 47, 237 (1981); S. Haroche and D. kleppner, Physics Today 42, 24 (1989).

7. B. R. Mollow, Phys. Rev. 188, 1969 (1969).

8. C. Cohen-Tannoudji, J. Dupont-Roc, and G. Grynberg, Atom-Photon Interactions (Willy, New York, 1992). 
9. W. E. Lamb and R. C. Retherford, phys. Rev. 79, 549 (1950); ibid. 81222 (1951).

10. H. A. Bethe and E. E. Salpetee, Quantum Mechanics of One- and Two- Electron Atoms (Springer Verlag, 1957).

11. A. P. Kazantsev, G. J.Surdutovich, and V. P. Yakovlev, Mechanical Action of Light on Atoms (World Scientific, Singapore 1990).

12. M. Wilkens, Phys. Rev. A 47, 671 (1993).

13. Gao-Jian zeng, Shi-Lun zhou, Sheng-Mei Ao, and Zhao-Yang Zeng, Phys. Rev. A 55, 2945 (1997).

14. L. Kouwenhoven and C. Marcus, Physics world, 35, June (1998).

\section{Figure Captions}

Fig. 1 Ac Stark split of energy levels of a damped two-level atom.

Fig. 2 Rabi oscillation (a) for strong coupling and damped exponential (b) for weak coupling of transition probability $\rho_{1}\left(P_{0}+\hbar k, t\right)$. 


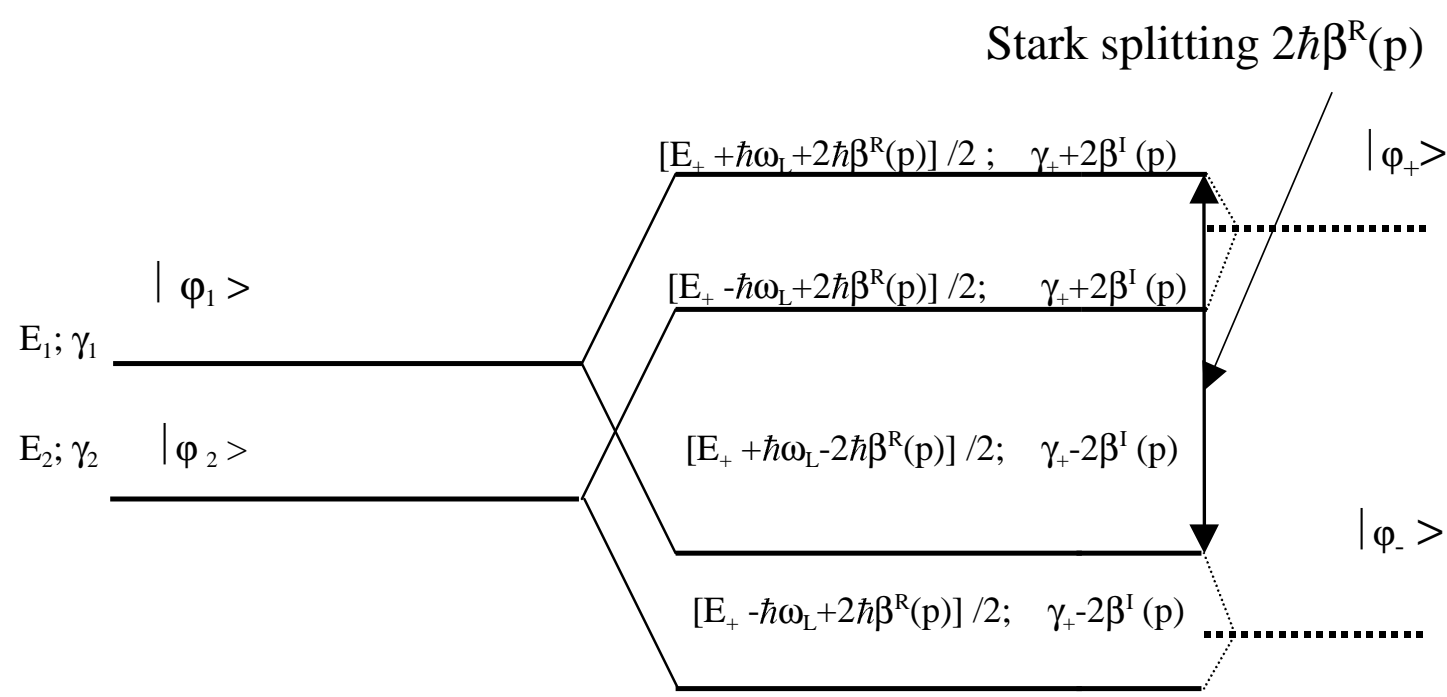

Fig. 1

Zeng et al. 
Fig. 2 Zeng et al.

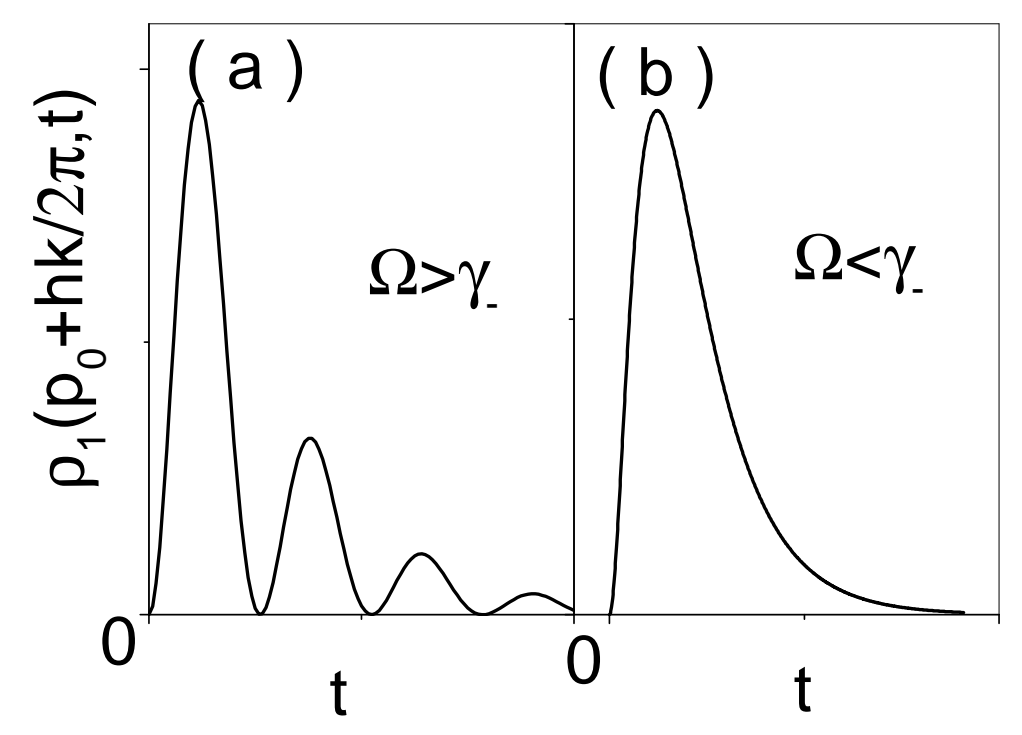

